\title{
Le discours électoral : entre transversalité et frontières génériques
}

Amadou Ouattara ADOU ${ }^{1}$

\begin{abstract}
The electoral speech appears as an asserted genre of political discourse with its own defining criteria. However there are speeches that are hardly part of an electoral context, but which carry relevant criteria of power conquest speeches. Our analysis of former Ivorian Prime Minister Amadou Gon Coulibaly's speech on the occasion of the Gôh region's day of tribute to President Alassane Ouattara is intended to challenge some defining features and thus, the generic boundaries of electoral discourse.
\end{abstract}

Keywords: electoral speech; generics boundaries; discourse processes; argumentative effects; transversality.

DOI: $10.24818 / D L G / 2021 / 38 / 05$

\section{Introduction}

$\mathrm{L}$

a quasi-totalité des travaux qui se sont intéressés à la communication politique l'inscrivent dans la perspective de la quête et de la gestion du pouvoir d'État. Cependant, même si de façon générale elle diffuse les idées et autres idéaux politiques et promeut les actes politiques, il faut relever que les discours qui en résulte sont classifiés en différents genres dotés de critères spécifiques. Par exemple, le tweet politique se distingue de l'Adresse à la nation pour des raisons que l'on peut retrouver dans leurs formes, dans les rhétoriques ou les modalisations mobilisées, etc. Aussi, force est de reconnaitre que les frontières qui délimitent les différents genres du discours politique ne sont pas totalement étanches. Mieux, certaines productions convergent, se télescopent ou s'imbriquent de telle sorte qu'elles deviennent difficilement différenciables. Il en va ainsi du discours électoral qui semble se retrouver à des moments ou dans des contextes qui ne lui sont pas dédiés. C'est ainsi à croire que tout discours reconnu politique, quel qu'en soit le contexte spatio-temporel, porte sur la

1 Amadou Ouattara Adou, Département de Lettres Modernes, Université Félix Houphouët-Boigny, Côte d'Ivoire, adouamed@yahoo.fr 
conquête ou la conservation du pouvoir. Il va sans dire que le discours électoral dont cette visée est l'apanage, "peut se mouler dans les autres genres du discours politique. » (Adou, Yao, 2020 : 189). Le problème de sa classification genrologique et de sa délimitation spatio-temporelle se pose donc avec acuité.

La présente contribution vise à requestionner les frontières génériques du discours électoral à l'effet de prouver son caractère transversal. Elle s'inscrit donc dans le cadre théorique des travaux portant sur l'argumentation dans le discours politique, et s'évertuera à analyser les procédés discursifs et les effets argumentatifs déployés dans une allocution de l'ancien Premier Ministre ivoirien, Amadou Gon Coulibaly (AGC), faite en dehors de tout contexte électoral. Pour ce faire, elle partira d'abord de la présentation de l'univers de référence qui sous-tend le dit discours.

\section{Univers de référence du corpus}

Le corpus que nous analysons dans cet article est constitué du discours de l'ancien Premier Ministre AGC, tenu le 12 janvier 2019 à Gagnoa dans le cadre de la Journée de reconnaissance, d'hommage et de soutien de la région du Gôh au Président de la République, Alassane Ouattara $(\mathrm{AO})^{2}$.

De fait, la journée organisée à Gagnoa s'inscrit dans la lignée des activités organisées par les responsables du Rassemblement des Houphouëtistes pour la Démocratie et la Paix (RHDP) ${ }^{3}$ des différentes régions de la Côte d'Ivoire en reconnaissance des actions de développement initiées par le Président $\mathrm{AO}$ en faveur des dites régions, depuis son accession à la magistrature suprême. Celle de Gagnoa succède à celle d'Oumé ${ }^{4}$ et a été organisée en début d'année. C'est dire que le cadre temporel n'est aucunement lié aux élections : les élections législatives ont eu lieu en 2016, les municipales et les régionales en 2018 et l'élection

\footnotetext{
${ }^{2}$ http://www.gouv.ci/doc/1579025658VISITE-DE-TRAVAIL-DANS-LA-REGIONDU-GOH-GAGNOA-ALLOCUTION-DE-PREMIER-MINISTRE-AMADOUGON-COULIBALY.pdf.

${ }^{3}$ Il s'agit du parti au pouvoir. Il est dirigé par le Président ivoirien, Alassane Ouattara.

${ }^{4}$ Cette ville du centre-ouest est le chef-lieu d'un département de la même région que Gagnoa : la région du Gôh.
} 
présidentielle est prévue pour octobre 2020, conformément à la constitution en vigueur.

Aussi, le contexte de production de ce discours le présente comme un discours épidictique :

"Discours cérémoniel de louange ou de blâme, le discours épidictique s'oppose au discours délibératif (...). Moins ouvertement politique que le genre délibératif, le genre épidictique semble préférer la contemplation à l'action, le consensus à la polémique. En réalité comme le montre Barbara Cassin, il utilise le moment opportun (kairos) de la cérémonie officielle à des fins politiques : Il s'agit de (re)construire le consensus et de mettre en valeur l'orateur en rappelant habilement, au moyen de lieux communs (topoï), les valeurs qui unissent la communauté. » (A La Guillaume Luc Bénoit, 2000 : 8).

Somme toute, le discours épidictique se caractérise par la louange ou le blâme d'une personnalité pour laquelle une cérémonie est organisée et a pour visée de distinguer le beau du laid. Dans le cas présent, il s'agit de la louange adressée au Président de la République, par des populations, pour les actes de développement posés ou initiés en leur faveur. En tant que contexte d'hommage, le cadre autorise des propos élogieux, voire même dithyrambiques de la part des locuteurs représentant les populations initiatrices de la cérémonie. A propos de la rhétorique épidictique, Aristote (1368: 114) note que «l'amplification est la mieux appropriée au genre épidictique, car il a pour matière des actions sur lesquelles tout le monde est d'accord ; il ne reste plus qu'à leur attribuer importance et beauté. »

Parallèlement, le contexte d'hommage autorise une expression de gratitude et éventuellement des promesses dans la continuité des actions ayant suscité la célébration, de la part du bénéficiaire ou éventuellement de son porte-parole.

\section{Procédés discursifs et effets argumentatifs}

Il est certes vrai que les différentes stratégies ou les différents arguments présenté(e)s dans les traités d'argumentation n'opèrent pas toujours et partout avec la même efficacité, mais l'observation des procédés mobilisés dans les discours de conquête de pouvoir permet d'établir certaines constantes comme structurant l'argumentation d'un discours électoral.

Coulibaly Nanourougo (2011: 3) note par exemple que dans le discours électoral, « le souci de l'énonciateur est d'élaborer et de présenter 
une image de lui qui réponde aux attentes du public, de tourner en dérision ses adversaires en démontrant l'inadéquation de leurs pensées et de leurs projets ». Dans cette même veine, Labbé Dominique et Monière Denis (2010 : 83) font remarquer que

"le discours électoral est plus ancré dans les problèmes du présent et contient plus de références à la communautë (...). C'est aussi un discours qui valorise le sens collectif (...). Le discours électoral est aussi moins abstrait et plus orienté vers l'action (...) La forte présence de la construction négative, de même que la désignation des adversaires soulignent le caractère polémique du discours électoral. »

De ce qui précède et des travaux portant sur le marketing politique (André, Claude, 2014) ou sur le meeting électoral (Haddad, Raphaël, 2017), l'on peut retenir que les arguments déployés dans ce genre de discours politique surfent sur les registres de l'ethos ou de l'image du candidat (construction, renforcement ou réhabilitation), de la disqualification de l'adversaire, de la promesse, etc. Il va sans dire que les stratégies de légitimation ${ }^{5}$ et de délégitimation, de crédibilisation et de décrédibilisation y sont très prégnantes.

\subsection{Des procédés discursifs mobilisés}

\subsubsection{La focalisation}

En linguistique, la focalisation désigne «une opération (souvent synonyme d'emphase) qui met en valeur un constituant de la phrase, ou focus.» (Maingueneau, Dominique, 2002: 265). Aussi, à la suite des travaux de Riegel Martin (1983) sur les focalisations contrastive et identificatrice et de Nolke Henning (2001) sur la focalisation énonciative, nous posons que la focalisation discursive désigne l'opération de présentation et de caractérisation des acteurs du discours dans un énoncé. Cette opération, qui relève du sujet parlant, induit des effets argumentatifs, comme l'ethos ou le pathos et s'appuie sur les indices de leur inscription énonciative. Toutefois, la particularité et la pertinence de l'opération de focalisation ici relève du fait que le locuteur procède, en plus des acteurs traditionnels du discours (locuteur et interlocuteur), à une mise en focus du délocuté.

${ }^{5}$ Charaudeau pense que le discours politique mobilise trois grandes stratégies: légitimation, crédibilité, captation (2014) 
Par ordre d'importance, nous notons dans le discours une focalisation axée sur l'auditoire, le locuteur et le délocuté, conformément au cadre communicationnel et aux enjeux en présence.

Il faut le rappeler, AGC produit son discours à l'occasion d'une cérémonie d'hommage au Président de la République, qu'il représente pour l'occasion. Le cadre scénique implique alors que les énoncés portent essentiellement sur le bénéficiaire de l'hommage et sur ses initiateurs. Il apparait donc logique que les indices relatifs à l'auditoire principal et immédiat, les hôtes du jour, connaissent une distribution numérique importante. L'on note par exemple pour l'auditoire, indifféremment de sa stratification, un total de 38 occurrences dont 19 emplois de vous (11) et de votre/vos (8) ; 15 emplois des GN, tels que «vaillantes populations de la grande région du Gôh, Mesdames et Messieurs, Jeunes de la région, tous les cadres, etc., et 5 usages des termes de parenté « chers frères et sœurs ».

En ce qui concerne les déictiques se référant au locuteur, il y a un total de 30 emplois : «Je» (23) ; «me» $(4)$; «mon/ma/mes» (3). Enfin, pour le délocuté, nous comptons 14 occurrences de la désignation nominale consacrée "SEM le Président de la République », accompagnée quelques fois de son nom «Alassane Ouattara», 4 emplois des possessifs «son/sa/ses » et 1 usage du pronom personnel «il»; soit un total de 19 occurrences.

Aussi, la focalisation de l'auditoire, ou du moins son adresse, ne procède pas de sa mise en scène, mais plutôt de sa prise en compte discursive modulée de sorte à réduire la distance énonciative putative et à instaurer une relation familière entre le sujet parlant et lui. A propos de la relation familière, Rigat Françoise $(2010: 8)$ note que

«Les formes d'adresse doivent non seulement exprimer la bienveillance, essentielle pour gagner la confiance de l'auditoire (Aristote, cité par Amossy, 1999, 61) mais aussi établir une connivence entre le candidat et l'électeur ou mieux, provoquer un effet de proximité, comme on dit dans le champ socio-politique. Sur un axe social, la dynamique de la proximité renvoie à une relation de solidarité, de rapprochement dù à l'appartenance au même groupe (Kerbrat-Orecchioni, 1992, 40) et à l'attachement au territoire dans lequel les candidats se présentent. »

Ainsi, si les désignations nominales «Les chefs traditionnels, Femmes de Gagnoa, Jeunes de la région, tous les cadres, Mesdames et Messieurs » apparaissent comme des indicateurs axiologiquement neutres, les termes de parenté "Chères sœurs, chers amis jeunes" sont énonciativement marqués et porteurs d'affectivité. Ils sont composés de 
l'adjectif épithète antéposé "chers/chères » qui "exprime un sentiment " selon le dictionnaire Trésor de la Langue Française en ligne, et des substantifs «sœurs et amis jeunes ». Ainsi, ces substantifs désignant deux auditoires spécifiques, les femmes d'une part et les jeunes d'autre part, sont déterminés par l'adjectif «cher» qui adopte alors une valeur particulière, celle d'annoncer un type de relation. En prenant en compte le fait que le sujet parlant et ses allocutaires n'aient pas la même filiation et n'entretiennent pas effectivement une amitié, l'on peut déduire que l'adjectif a un rôle renforçatif des relations fraternelle et amicale établies de fait par la convocation de ces substantifs. Ces termes d'adresse apparaissent alors comme des termes relationnels. D'ailleurs, pour Cathérine Kerbrat-Orecchioni (2010:27), «C'est en grande partie sur les termes d'adresse que repose la gestion de la relation interpersonnelle, que ces formes peuvent confirmer, modifier ou constituer ». La distance énonciative instaurée et entretenue par l'absence de commerce relationnel habituel entre les protagonistes de l'interaction s'en trouve ainsi réduite, laissant place à une proximité, tout au moins discursive. L'objectif de l'instauration par l'orateur de cette relation proxémique est sans doute de créer une connivence entre lui et cet auditoire à l'effet de faire accepter son propos sur l'objet: le délocuté. Et c'est à ce niveau que la focalisation du délocuté est pertinente.

De fait, les termes de désignation de $\mathrm{AO}$ montrent un respect de l'us protocolaire en matière de désignation des présidents de République : Son Excellence Monsieur le Président de la République. La distribution de ce terme peut alors indiquer sa primauté sur les adjectifs possessifs «son/sa/ses » et le pronom personnel «il ». Mais à côté de ces désignations, l'orateur convoque des évaluatifs axiologiques portant sur les actes posés par le président Alassane Ouattara en faveur de la région du Gôh et des autres régions du pays, lesquels amplifient la focalisation énonciative visée : «ce grand homme d'État», "sa grande œuvre de construction d'une nation prospère, fraternelle et en paix », « une véritable chance (...) pour le développement», «sa ferme et constante volonté de construire une Côte d'Ivoire nouvelle, moderne et prospère ", "son action de paix et de développement », "bilan particulièrement élogieux de sa gouvernance », "les importants investissements», "les investissements massifs», "ces investissements importants de développement», "sous la haute égide du Président ». Le caractère épidictique du discours induit par la nature de l'évènement qui l'occasionne, autorise des qualificatifs élogieux à l'endroit 
du célébré. Toutefois, la mobilisation autour de la personne du délocuté, du fait de son bilan de gouvernance qualifié de «bilan particulièrement élogieux» par le locuteur pourrait faire penser à discours de campagne électorale.

Aussi, à la conclusion de son discours, AGC dit: "Je voudrais lancer ici à Gagnoa à nouveau, un appel au rassemblement et à la cohésion autour du Président de la République, SEM Alassane Ouattara. Ensemble, poursuivons, dans la cohésion, la rigueur et le travail, nos efforts pour construire, sous la haute égide du Président de la République, SEM Alassane Ouattara, une Côte d'Ivoire de progrès continu, de paix et de bonheur pour nous-mêmes, nos enfants et petits-enfants. »

En effet, la répétition des termes consacrés SEM et Président de la République n'est pas sans renforcer la mise en focus du désigné, en plus de l'invitation faite à l'auditoire de se rassembler autour de ce dernier à travers la modalité intersubjective présente dans cet énoncé (Le Querler, Nicole, 1996: 133). Cette mobilisation souhaitée est justifiée par la construction d'une "Côte d'Ivoire de progrès continu, de paix et de bonheur» pour la postérité. Cette formule (Krieg-Planque, Alice, 2009) s'apparente au résumé d'un projet de société présenté à l'auditoire en vue de solliciter son suffrage. Le rassemblement et la cohésion autour du président sollicités par le sujet parlant peuvent se justifier soit par la nécessité de conserver les acquis et de continuer à le soutenir pour le temps qui reste avant l'élection (22 mois), soit pour le ou les autres mandats à venir. Dans le premier cas, la souscription demandée sera profitable d'une part au président avant la fin de son mandat, et d'autre part au candidat qu'il proposera en tant que président du RHDP, au cas où il ne serait pas candidat lui-même. Dans le deuxième cas, elle lui sera bénéfique jusqu'à ce qu'il quitte le pouvoir. Dans tous les cas, cette mobilisation est requise pour le candidat du régime, comptable des bonnes actions célébrées. Nous percevons alors l'importance de la formule résumative citée supra.

De même, le projet de société peut être entendu comme l'idéal de société visé par un candidat, qui se décline en programme de gouvernement ou actes de gouvernement. Lors de la cérémonie de présentation du projet du candidat du RHDP à la présidentielle d'octobre 2020, Alassane Ouattara, organisée le 15 octobre 2020, le Directeur National de campagne ${ }^{6}$ dira :

${ }^{6}$ Le ministre Patrick ACHI, Secrétaire Général de la Présidence.

Dialogos • Vol. XXII No. 38/2021 
"Les cinq piliers de notre programme 2021-2025, se déclinent comme suit :

1 La consolidation de la paix, de la sécurité et de la cohésion sociale;

2 La création de richesse, source d'emplois ;

3 La création d'un cadre de vie agréable et d'un environnement attractif de nos régions ;

4 Le développement d'une administration plus performante et plus engagée ;

5 L'Ivoirien, en tant qu'acteur de développement, au cour de la Cote d'Ivoire solidaire. $»^{7}$

Diverses interprétations peuvent être faites de ce programme, mais force est de constater qu'il peut être condensé dans la formule dite par le sujet parlant aux populations, faisant office de fondement à son appel. D'ailleurs, que la formule prononcée par le sujet parlant soit la reformulation du slogan du candidat Alassane Ouattara à l'élection d'octobre 2020, laquelle est porteuse de son projet: "La Côte d'Ivoire solidaire », ou une formule produite pour la circonstance, il n'est pas faux de dire qu'elle cadre avec un contexte électoral ou plus précisément une situation de meeting électoral où le candidat est mis en focus. Dans un meeting électoral, le candidat ou ses représentants mettent en avant l'ensemble des actions déjà menées à l'effet de projeter de lui un ethos de compétence et d'inviter l'auditoire-électorat à s'inscrire dans la continuité en l'élisant. C'est dans cette perspective que semble s'inscrire la présentation de son bilan de gestion qui procède d'une narration.

\subsubsection{La narration}

Dans son usage rhétorique, la narration renvoie à l'étape de présentation des faits, dans la structure aristotélicienne de l'argumentation. Baron (1849: 130) note à ce propos que «la narration est l'exposé des faits ». De même, pour Patrick Charaudeau (2014: 26), «la narration consiste à décrire les «faire » de ces êtres (les actions qu'ils accomplissent ou subissent et les évènements dans lesquels ils sont pris), à l'aide de divers ,l.exprime sa gratitude, sa volonté de continuer à poser des actes du même registre, ou qu'il présente les actes à venir. Mais que le représentant, porteparole du célébré, rappelle ces faits, cela laisse soupçonner une dynamique électorale, surtout que le contexte et le discours sont politiques. Une telle

${ }^{7}$ www.ado2020.ci, consulté le 17 février 2021. 
dynamique repose nécessairement sur la modélisation (Sallaberry, 2005) opérée par AGC. De cette modélisation, l'on peut inférer que AGC se pose comme locuteur représentant et porte-parole du président, et pose l'auditoire d'une part comme les interlocuteurs organisateurs de la journée d'hommage, et d'autre part comme un électorat qu'il faut rallier à la cause $\mathrm{du}$ RHDP. Les remerciements de départ justifient la première image de l'auditoire et le rappel du bilan et l'appel à la mobilisation, la seconde. A partir de ce moment la narration des investissements vient en appoint à l'argumentation dont la substance peut être ainsi formulée : mobilisez-vous autour du président Alassane Ouattara. La finalité de cette mobilisation pourrait être recherchée dans la perspective électoraliste. Les effets argumentatifs produits par la focalisation et la narration peuvent confirmer cette hypothèse.

\subsubsection{Des effets argumentatifs}

3.1.3.1 L'argument du gaspillage

L'argument dit $\mathrm{du}$ gaspillage est un argument ou un fait d'argument qui découle de l'exposé de faits ou d'actions avec pour effet de considérer les sacrifices consentis dans l'accomplissement de ces actions et de ne pas en perdre le bénéfice par de mauvais choix. En d'autres termes, les bénéficiaires d'une action ne doivent pas, par un quelconque choix, mettre un terme aux efforts fournis pour accomplir cette action, sinon les sacrifices consentis deviendraient du gaspillage. Pour Robrieux JeanJacques (1993:184), vu qu'« un sacrifice a déjà été consenti: il faut poursuivre l'opération sous peine d'en perdre le bénéfice et de se voir taxé d'incohérence. »

Le sacrifice consenti ici est à voir dans les différents investissements rappelés, lesquels ont été réalisés par le président Alassane Ouattara dans l'optique d'améliorer les conditions de vie des populations de cette région. Il est également et surtout à déduire du propos conclusif de la présentation $\mathrm{du}$ bilan: " $\mathrm{Au}$ regard de ces investissements importants de développement dans cette région en particulier, et au plan national en général, il est de notre responsabilité, à tous, de préserver la paix si durement acquise et de maintenir la Côte d'Ivoire dans le cercle vertueux dans lequel elle se trouve désormais. »

La modalisation effectuée dans cet énoncé montre la convocation de deux subjectivèmes évaluatifs permettant au sujet parlant de porter un 
jugement de valeur sur l'objet de son propos: l'adjectif «importants » et l'adverbe "durement». Le premier implique que les investissements ne sont pas négligeables tandis que le second porte sur la paix qui n'aurait pas été acquise avec aisance. De même, une opération de généralisation manifestée par l'usage des locutions «en particulier » et «en général » permet d'étendre le bénéfice des investissements à toutes les régions du pays. Il en ressort dans un premier temps, que les importants investissements réalisés par le président et son gouvernement ont permis d'instaurer un climat de paix dans toutes les régions du pays. Cet état de fait est renforcé par l'adverbe évaluatif «désormais » dont le présupposé est que le pays n'était pas dans le cercle vertueux désigné. Il est donc logique qu'ils soient félicités. Dans un second temps, la structure syntaxique de l'énoncé informe primo que la paix acquise et l'inscription du pays dans le cercle vertueux sont liées aux investissements réalisés et secundo, que ces acquis doivent être maintenus par « responsabilité ». De fait, la séquence « au regard de ces investissements ... en général » apparait comme le complément circonstanciel de cause de la proposition principale «il est de notre responsabilité à tous » et des complétives infinitives «de préserver ... le cercle vertueux». La relative «dans lequel il se trouve désormais » précise le cercle dont il s'agit. Somme toute, le lien de causalité entre les actes de développement posés et la paix et ses corolaires, justifie la présence de l'argument de gaspillage. L'idée de sacrifice est portée par « les investissements importants de développement» et renforcée par «durement» et celle de gaspillage réside dans la possibilité de ne pas en préserver les fruits (paix et cercle vertueux). La responsabilité convoquée comme topos ici est alors justifiée dans la mesure où le bon sens recommande que les acquis, surtout bénéfiques, soient préservés par tous. Cette implication résonne en écho à l'appel à la mobilisation lancé en fin d'allocution. Ainsi, à en croire l'orateur, c'est surtout par responsabilité ou par devoir que son auditoire, mais également tous les Ivoiriens adressés par trope communicationnel (notre responsabilité à tous), doivent accompagner le président dans sa dynamique de développement. Si cet accompagnement venait à manquer, les efforts consentis seraient vains et le pays pourrait sortir $\mathrm{du}$ cercle vertueux dans lequel il se trouve « désormais ». Il s'agit là de la rhétorique de la continuité, qui plus est, est une stratégie fortement mobilisée dans le discours électoral. Autrement dit, AGC semble mobiliser des électeurs à continuer de faire confiance au président ou à son régime (le RHDP), qui aura certainement un candidat à 
la prochaine élection présidentielle. La doxa selon laquelle l'on ne change pas une équipe qui gagne est mobilisée implicitement comme valeur partagée ou vérité admise au même titre que la responsabilité de préserver la paix. Or la continuité ou son antonyme la rupture n'intervient que dans la possibilité du changement. Dans le domaine politique, il ne peut s'agir que $\mathrm{du}$ cadre électoral où la possibilité est donnée aux populations/électeurs de renouveler leur confiance en l'équipe dirigeante ou de la changer en élisant une nouvelle.

\subsubsection{La stratégie de crédibilité}

A propos de la crédibilité, Patrick Charaudeau (1998:14) note qu'elle «est un fait de stratégie de discours qui (...) consiste pour le sujet parlant à déterminer une position de vérité, de sorte qu'il puisse (...) être pris au sérieux ». Il la situe, avec la légitimation et la captation, parmi les stratégies discursives les plus mobilisées par les orateurs politiques. Nous la posons ici comme une finalité ou un effet argumentatif visée dans le discours électoral dans la mesure où le suffrage demandé à l'auditoireélectorat est censé se fonder sur la confiance établie entre le candidat et l'électorat. Or la crédibilité est le socle de la confiance. Il va sans dire que c'est parce que l'orateur-candidat aura réussi à produire une image de crédibilité que l'auditoire-électorat décidera, par la voie des urnes, de lui confier sa destinée. De même, la grande majorité des arguments déployés dans le discours électoral prennent appui sur l'effet de crédibilité s'il est déjà acquis ou participe de sa production s'il n'est pas encore acquis. C'est ce qui justifie par exemple que le candidat se montre le plus apte ou le plus compétent, dénigre son ou ses adversaires et s'autorise des promesses, dans le discours électoral.

Ici, cet effet de crédibilité pourrait être perçu à deux niveaux : la crédibilité du locuteur (AGC) et celle de l'énonciateur (AO).

En effet, à partir du moment où il est le représentant du célébré, nous posons avec Maingueneau Dominique (2012: 144) que l'énonciation s'opère sur un double plan, mais avec le même auditoire et les mêmes visées. AGC est le locuteur, c'est-à-dire celui à qui réfèrent les déictiques de la première personne dans ce discours. Le président, l'objet de la célébration, est l'énonciateur dans la mesure où le locuteur est son porteparole. Il est discursivement responsable des contenus propositionnels délivrés par l'orateur, d'autant plus qu'au sens littéral, c'est sa parole qui est portée. Elle peut avoir été portée textuellement ou avec des 
modifications, mais toujours est-il qu'il est soit informé et donc en accord avec le discours dont son représentant est porteur, soit qu'il l'assume sur la base de la confiance établie entre lui et son représentant. De ce fait, la crédibilité peut être au profit du locuteur qui chercherait à prouver à l'auditoire qu'il est effectivement l'un des hommes de confiance du président de la République et que sa parole est par ricochet crédible. Elle peut être également au profit de l'énonciateur qui chercherait à prouver qu'il est crédible et par ricochet autorisé à dire ou à demander ce que le locuteur dit ou demande en son nom.

Aussi, eu égard au contexte socio-communicationnel, la primauté est accordée à la crédibilité visée en faveur de l'énonciateur, le délocuté de AGC.

En effet, la présentation du bilan de gestion de $\mathrm{AO}$, telle qu'analysée dans la narration ainsi que dans l'argument du sacrifice, vise également à produire un effet de crédibilité à l'actif de ce dernier qui passe par la construction d'une image de président compétent et soucieux du bien-être des populations.

La construction de l'image de président compétent peut se justifier implicitement par la conception des différents projets de développement et à la mobilisation des moyens y afférents. Pour rappel, l'adverbe temporel «désormais» qui modalise "le cercle vertueux dans lequel le pays se trouve » laisse présupposer que le pays n'était pas dans la situation mirobolante, ou du moins améliorée, dans laquelle il se retrouve grâce à l'action du président $\mathrm{AO}$ et de son gouvernement. Cela pourrait sousentendre que les anciens présidents et leurs gouvernements n'avaient pas réussi à faire entrer le pays dans ce cercle. A partir de ce moment, le génie créateur, l'expertise et la compétence du président actuel est à reconnaitre et même à saluer. N'est-ce d'ailleurs pas l'objectif de la cérémonie d'hommage organisée en son honneur? Le propos du locuteur vient alors en confirmation de l'image de compétent préexistante dans l'esprit des organisateurs et de l'auditoire acquis à sa cause. De plus, il faut souligner le fait que ces actes de développement ne se limitent pas à cette seule région ; ils s'étendent à toute la Côte d'Ivoire : "Au regard de ces investissements importants de développement dans cette région en particulier, et au plan national en général», "travailler au bonheur des populations, à la grandeur de la Côte d'Ivoire", "l'œuvre de construction de la Côte d'Ivoire ». Ces différents énoncés convoqués pour indiquer l'impact des actes du président, informent en plus de l'espace que couvrent ces actes. 
Mais ils s'appuient sur le présupposé que le président a le souci du bienêtre de ses administrés, mais également qu'il a la compétence de la mobilisation des moyens nécessaires à l'atteinte de cet objectif. Autrement dit, s'il n'avait pas à cœur d'améliorer la vie des populations vivant en Côte d'Ivoire et la possibilité (connaissances et compétences nécessaires) de trouver les ressources humaines et financières pour l'exécution des travaux, les actes rappelés et/ou annoncés ne pourraient l'être. De même, cette image discursive ainsi construite ou confirmée est mise en orbite par la comparaison qu'induit "désormais", laquelle comparaison oppose le président Alassane à tous ses prédécesseurs. Il apparait alors comme un président plus compétent que les autres, ou mieux, comme le président compétent vu que c'est justement lui qui a réussi à inscrire le pays dans ce cercle vertueux. Si cet argument opère, le résultat logique attendu est que les populations du Gôh et par-delà, de toutes les régions ivoiriennes, s'inscrivent dans la continuité. Le locuteur leur demande de continuer à faire confiance au président. Il peut alors lancer, en fin de discours : «Ensemble, poursuivons (...) nos efforts pour construire, sous la haute égide du Président de la République, SEM Alassane Ouattara, une Côte d'Ivoire de progrès continu ».

\section{Conclusion}

Par l'analyse des procédés de focalisation et de narration discursives tels que convoqués dans ce discours de l'ancien Premier Ministre ivoirien, de même que les effets argumentatifs que ces procédés produisent, nous retenons que les frontières génériques qui séparent le discours électoral des autres genres du discours politique sont relativement poreuses.

Aussi, est-il possible de noter que les caractérisations ou traits définitoires qui lui sont attribuées jusque-là, ne sont pas de son seul apanage. Certains discours produits en dehors de tout contexte électoral, à l'image de celui analysé ici, apparaissent sous des traits de discours de conquête du pouvoir. Relèvent-ils du discours préélectoral ? D'ailleurs, quelle(s) différence(s) peut-on établir entre le discours électoral et le discours préélectoral? 


\section{Bibliographie}

1. A LA GUILLAUME, Luc Benoit (2000), Les discours d'investiture des présidents américains ou les paradoxes de l'éloge, L'Harmattan, Paris.

2. ADOU, Amadou Ouattara et YAO, Kouadio Raymond (2020), « Le discours de conquête du pouvoir politique et la question des genres: étude de cas dans l'adresse à la nation de Guillaume SORO » in Le discours de conquête du pouvoir politique en contexte africain, Revue SLADI, n², Bouaké, Editions SLADI, p. 175-190.

3. AMOSSY, Ruth (1999), Images de soi dans le discours. La construction de l'ethos, Nathan, Paris.

4. ANDRE, Claude (2014), Essai sur le marketing politique et les stratégies gouvernementales au Québec : réflexions critiques, Mémoire de Maitrise de Science politique, Université du Québec à Montréal, Montréal.

5. ARISTOTE (1991), Rhétorique, UGE, coll. « Le Livre de Poche », Paris.

6. CHARAUDEAU, Patrick (2005), Le discours politique. Les masques du pouvoir, Vuibert, Paris.

7. CHARAUDEAU, Patrick (2005), «Quand l'argumentation n'est que visée persuasive. Exemple du discours politique » in Burger M. et Martiel G., Argumentation et communication dans les médias, Québec, Nota Bene, p. 23-43.

8. CHARAUDEAU, Patrick (1998), «L'argumentation n'est pas ce que l'on croit» in RevueLe français d'aujourd'hui, n²123, Association française des Enseignants de français, Paris.

9. CHARAUDEAU, Patrick et MAINGUENEAU, Dominique (2002), Dictionnaire d'analyse du discours, Seuil, Paris.

10. COULIBALY, Nanourougo (2011), «Manœuvres de persuasion électoralistes dans les discours de Laurent Gbagbo» in Revue Interstudia in revue Interstudia, « Feindre, mentir, manipuler. Culture et discours de la mystification», Revue du Centre Interdisciplinaire d'étude des formes discursives contemporaine Interstud, $\mathrm{N}^{\circ} 8, \mathrm{Bacau}, \mathrm{p}$. 198-208.

11. HADDAD, Raphaèl (2017), Le discours de meeting électoral : rituel d'affrontement, médiatisations, communication politique. Analyse du discours de meeting électoral pour l'élection présidentielle française (2002, 2007, 2012), Thèse de Doctorat, Linguistique. Université ParisEst. 
12. KERBRAT-ORECCHIONI, Cathérine (1992), Les interactions verbales, Tome II, Armand Colin, Paris.

13. KERBRAT-ORECCHIONI, Catherine (2010), «Introduction» in S'adresser à autrui. Les formes nominales d’adresse en français, Chambery, Éditions de l'université de Savoie.

14. KRIEG-PLANQUE, Alice (2009), La notion de «formule » en Analyse du discours, Presses Universitaires de Franche-Comté, Besançon.

15. LABBE, Dominique et MONIERE, Denis (2010), "Quelle est la spécificité des discours électoraux? Le cas de Stephen Harper» in Revue canadienne de science politique, Cambridge University Press (CUP), n43, vol. 1, Cambridge, p. 69-86.

16. LE QUERLER, Nicole (1996), Typologie des modalités, Presses Universitaires de Caen, Caen.

17. MAINGUENEAU, Dominique (2012), Analyser les textes de communication, Armand Colin, Paris.

18. NOLKE, Henning (2001), Le regard du locuteur 2. Pour une linguistique des traces énonciatives, Editions Kimé, Paris.

19. RIGAT, Françoise (2010), " Mes chers compatriotes »: stratégies discursives de l'interpellation des électeurs dans les professions de foi » in Corela [En ligne], HS-8, L'interpellation.

20. ROBRIEUX, Jean-Jacques (1993), Eléments de rhétorique et d'argumentation, Dunod, Paris

21. SALLABERRY, Jean-Claude (2005), «La représentation et le geste. Pragmatique et représentation ", in Spirale. Revue de recherches en éducation, hors-série $n^{\circ} 4,2005$. Les représentations en formation (2), pp. 5-33.

22. www.ado2020.ci

23. www.gouv.ci 